\title{
Thermal mismatch stress relaxation via lateral epitaxy in selectively grown GaN structures
}

\author{
Tsvetanka S. Zheleva, ${ }^{\mathrm{a}, \mathrm{b})}$ Waeil M. Ashmawi, ${ }^{\mathrm{c}}$ ) Ok-Hyun Nam, and Robert F. Davis \\ Department of Materials Science and Engineering, North Carolina State University, Raleigh, \\ North Carolina 27695-7907
}

(Received 14 September 1998; accepted for publication 12 February 1999)

\begin{abstract}
A reduction in the dislocation density of $10^{4}-10^{5} \mathrm{~cm}^{-2}$ has been achieved via lateral epitaxial overgrowth (LEO) of GaN films selectively grown from stripes etched in $\mathrm{SiO}_{2}$ masks deposited on $\mathrm{GaN} / \mathrm{AlN} / 6 \mathrm{H}-\mathrm{SiC}(0001)$ heterostructures. The magnitudes and distribution of stresses generated in the LEO GaN layer and the $\mathrm{SiO}_{2}$, due primarily to differences in the coefficients of thermal expansion, were modeled using finite element (FE) analysis. These calculations showed that localized compressive stress fields of $\approx 3 \mathrm{GPa}$ occurred at the edges of the LEO GaN in the vicinity of the $\mathrm{GaN} / \mathrm{SiO}_{2}$ interface. Localized compression along the $\mathrm{GaN}$ substrate/ $/ \mathrm{SiO}_{2}$ interface and tension along the $\langle 0001\rangle$ direction were responsible for the change in shape of the $\mathrm{SiO}_{2}$ stripes from rectangular with flat sides to an airfoil shape with curved sides. The FE calculations also revealed that an increase in the width of the $\mathrm{LEO} \mathrm{GaN} \mathrm{regions} \mathrm{over} \mathrm{the} \mathrm{SiO}_{2}$ or the reduction in the separation between the GaN stripes (all other dimensions being fixed) resulted in a slight reduction in the compressive stresses along the $\mathrm{LEO} \mathrm{GaN} / \mathrm{SiO}_{2}$ interface and an increase in the compressive stress along [0001]. An increase in the shear stress, at the corners of the LEO GaN near the LEO GaN/ $\mathrm{SiO}_{2}$ interface, with an increase in the width of the LEO GaN region were also indicated. (C) 1999 American Institute of Physics. [S0003-6951(99)02715-1]
\end{abstract}

The recent advancements in GaN-based laser diodes by Nakamura et al. ${ }^{1}$ were achieved in part due to the use of the lateral epitaxial overgrowth (LEO) technique reported earlier for GaN films by several investigators using sapphire ${ }^{2-5}$ and on $6 \mathrm{H}-\mathrm{SiC}^{6-11}$ substrates. Transmission electron microscopy (TEM) studies have been conducted by Zheleva et al. ${ }^{8,10,11}$ on $\mathrm{GaN}$ pyramids and stripes selectively grown from circular openings and rectangular windows, respectively, contained within $\mathrm{SiO}_{2}$ masks deposited on $\mathrm{GaN} / \mathrm{AlN} / 6 \mathrm{H}-\mathrm{SiC}(0001)$ heterostructures. This research revealed that the volumes of the pyramids and stripes which laterally overgrew the masks contained $<10^{4}-10^{5}$ dislocations $/ \mathrm{cm}^{2}$. This was in contrast to the regions of vertical growth within the $\mathrm{SiO}_{2}$ windows which exhibited a density of threading dislocations of $10^{9}-10^{10} \mathrm{~cm}^{-2} \cdot{ }^{2,9-11}$ The crystallographic templates for lateral epitaxy of $\mathrm{GaN}$ are the $\{11 \overline{2} 0\}$ side facets when the $\mathrm{SiO}_{2}$ stripes are oriented along $\langle 1 \overline{1} 00\rangle$, and the $\{1 \overline{1} 00\}$ and $\{1 \overline{1} 01\}$ side $\mathrm{GaN}$ planes if the stripe windows are oriented along $\langle 11 \overline{2} 0\rangle$ direction. ${ }^{8,11}$ The vertical growth direction in both cases is $\langle 0001\rangle$.

Stress/strain fields in a heterostructure consisting of LEO $\mathrm{GaN}$ deposited within two striped windows in a $\mathrm{SiO}_{2}$ mask, the latter deposited on a GaN/AlN/6H-SiC(0001) substrate, were modeled using two-dimensional (2D) finite element analysis (FEA), in-plane strain conditions and the code ANSYS 5.3. ${ }^{12}$ Two units of the heterostructure being analyzed

\footnotetext{
a) Author to whom correspondence should be addressed; electronic mail: Tsvetanka_Zheleva@ncsu.edu

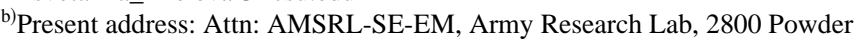
Mill Rd., Adelphi, MD 20783.

c) Also with: Department of Mechanical Engineering, North Carolina State University, Box 7907, Raleigh, NC 27695-7907.
}

were discretized using a quadratic four node element. The heterostructure was modeled within the framework of the continuum linear elasticity theory (Hook's law is valid, and hence the stress and strain tensors are linearly related via the elasticity tensor). The boundary conditions were such that all nodes on the side faces of the model were fixed against displacements in the respective directions of the surface normal. The nodes on the bottom of the $6 \mathrm{H}-\mathrm{SiC}$ substrate were fixed against displacements in the $\mathrm{z}$ direction to avoid rigid body shift. Elastic constants, Poisson's constants, coefficients of thermal expansion, density and the temperatures of the initial and final state were used as input parameters. ${ }^{13-15}$ The thermal load was applied considering the mismatches in thermal expansion coefficients without taking into account the lattice mismatches among the phases in the heterostructure. Room temperature was chosen as the reference temperature and the growth temperature for the LEO GaN $\left(1100^{\circ} \mathrm{C}\right)$ was chosen for the thermal load temperature. Various geometrical factors played important roles regarding the final thermal stress/ strain field distribution and hence the final deformed shape in the heterostructure: (i) the width and the thickness of the GaN stripes, (ii) the openings in the $\mathrm{SiO}_{2}$ windows, (iii) the $\mathrm{SiO}_{2}$ film thickness, (iv) the thickness of the underlying $\mathrm{GaN}$, and (v) the thickness of the AlN. In this study, the variation of the thermal stress as a function of the lateral growth distance, i.e., the width of the GaN stripes at a fixed window size was considered. A 2D, static model was employed. Though simple, this model described closely the morphology of the LEO GaN structures repeatedly observed by TEM.

A typical cross-sectional TEM image of a GaN stripe is shown in Fig. 1. As noted above, the density of threading dislocations is four to five orders of magnitude lower in the 


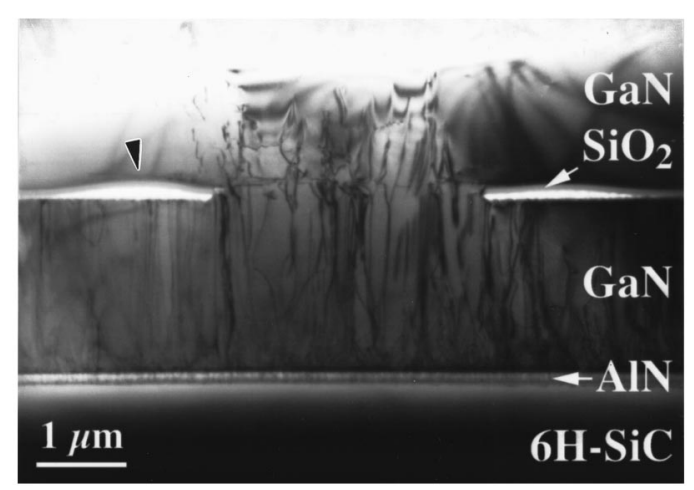

FIG. 1. Cross-sectional TEM of laterally overgrown GaN stripe in [1 $1 \overline{1} 00]$ orientation of the foil. The region in the selectively grown GaN above the $\mathrm{SiO}_{2}$ window has the same density of threading dislocations as the underlying $\mathrm{GaN}$ film; no dislocations were observed in the laterally grown $\mathrm{GaN}$ within regions above the $\mathrm{SiO}_{2}$.

laterally overgrown regions compared to that in the $\mathrm{GaN}$ material within the $\mathrm{SiO}_{2}$ window. The airfoil shape of the LEO GaN, pointed to by the arrow in Fig. 1, formed in response either to the thermal stresses generated on heating prior to deposition of the LEO GaN and due to the differences in the coefficients of thermal expansion of the underlying $\mathrm{GaN}$ and $\mathrm{SiO}_{2}$, or after the vertical growth of the $\mathrm{GaN}$ from the $\mathrm{SiO}_{2}$ windows, followed by lateral overgrowth. Some of the observed coalesced regions contain voids of different shape including drop-like, key-hole-like or triangular, as shown in Fig. 2(a). If the LEO GaN film is sufficiently thick, these voids terminate within this volume of the film. Above the voids, the volume of coalescence is usually without observable dislocations.

The stress-distribution results from the FE calculations are shown in Figs. 3(a)-3(c) and based on the assumption of

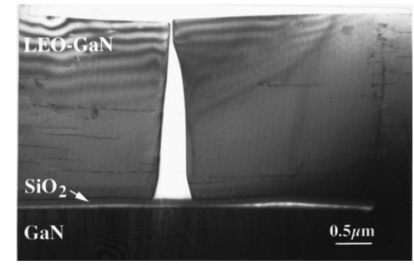

(a)

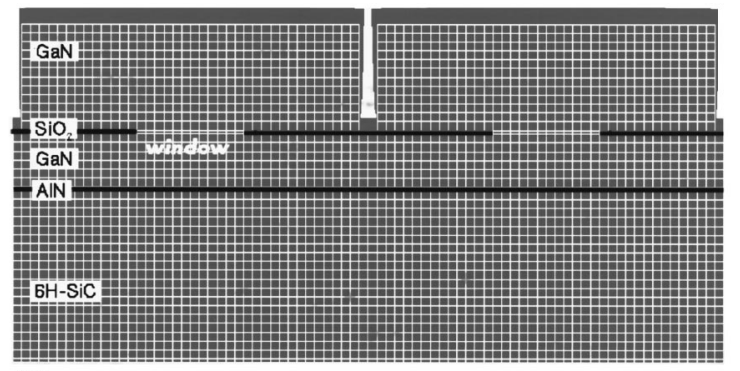

(b)

FIG. 2. TEM cross-sectional micrograph from a region of coalescence of LEO GaN stripes in [1 $1 \overline{1} 00]$ orientation of the foil. Please note that the bending of the LEO $\mathrm{GaN} / \mathrm{SiO}_{2}$ on the right side is more pronounced and there are fewer dislocations compared to the left side LEO GaN. (b) A schematic of the FEA model of the selectively grown $\mathrm{GaN}$ on $\mathrm{SiO}_{2}$ on $\mathrm{GaN} / \mathrm{AlN} / 6 \mathrm{H}-\mathrm{SiC}$ heterostructure used for our calculations. The mesh represents the initial state of discretization. The black bands outside the mesh represent the deformed shape after the thermal stress $\left(1100^{\circ} \mathrm{C}\right)$ is applied. represent the deformed shape after the thermal stress $\left(1100^{\circ} \mathrm{C}\right)$ is applied. lescence.

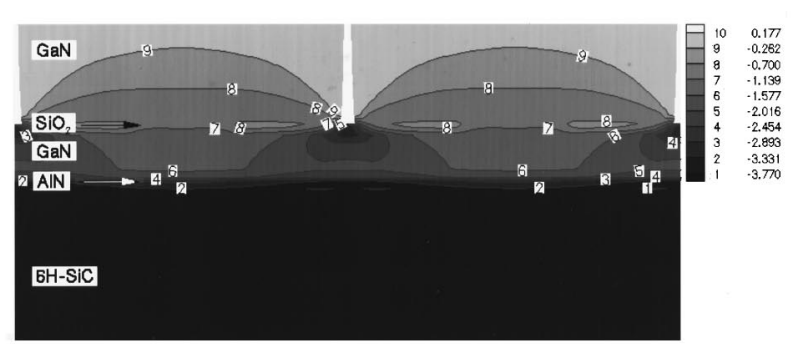

(a)

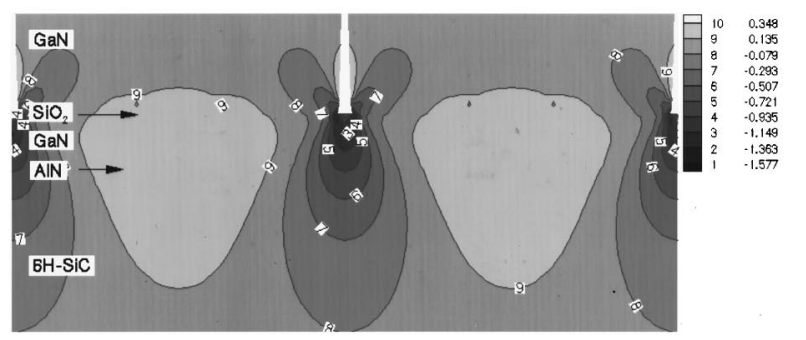

(b)

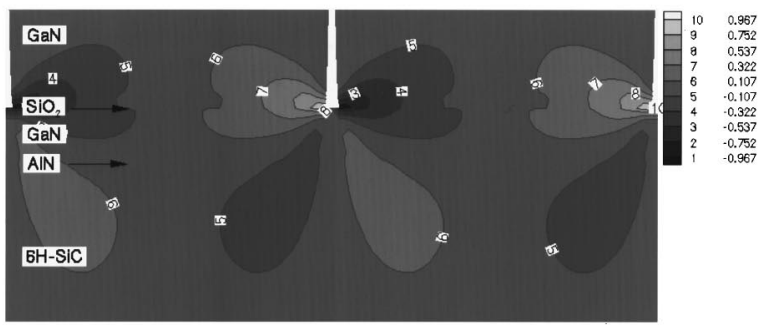

(c)

FIG. 3. Distributions of thermal stress fields (in GPa): (a) along the $x$ direction corresponding to the interface line along the $\langle 11 \overline{2} 0\rangle$ direction; (b) along the [0001] direction, or the vertical with respect to the interface planes, and (c) shear stresses shown as isocountour bands.

heating the complex LEO structure from room temperature to $1100{ }^{\circ} \mathrm{C}$. The minimum stress (maximum stress relaxation) in each of the GaN stripes is along the boundary between the tensile and compressive fields. The cross-sectional shape of the GaN stripe follows approximately the variation in the stress fields, i.e., the profiles in the stress gradients. It is known that the stresses in a heterostructure can be accommodated by two mechanisms: mechanical deformation and/or defect generation and propagation. It is evident from the FE analysis that stresses are generated as a result of the mismatch in the coefficients of thermal expansion among the phases in these heterostructures, which cause bending in the areas of lateral epitaxy, as shown in Figs. 1 and 2(a). The observed shape of these deformed areas and the corresponding thermally generated stress/strain profiles determined by FEA resemble the morphologies observed for many laterally grown GaN structures. Characteristics of the temperaturedependent stress profiles include: (i) localization of the $\sim 3$ GPa maximum compressive stress at the corners of the $\mathrm{GaN}$ stripes along the $x$ direction at the $\mathrm{GaN} / \mathrm{SiO}_{2}$ interface line [Fig. 3(a)], (ii) smaller localized compressive stress fields of $\sim 1.5 \mathrm{GPa}$ at the corners of the GaN stripes along the $\langle 0001\rangle$ direction, [Fig. 3(b)], and (iii) localized shear stresses of $\sim 0.9 \mathrm{GPa}$ at the LEO GaN edges [Fig. 3(c)]. These stresses in tandem with the tensile stress of $\sim 0.3 \mathrm{GPa}$ along the side edges of the LEO GaN cause the deformation observed at the side faces of the GaN stripes. This deformation determines the formation and shape of the voids in the regions of coa-

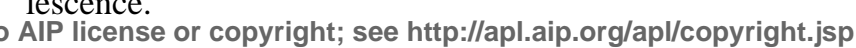




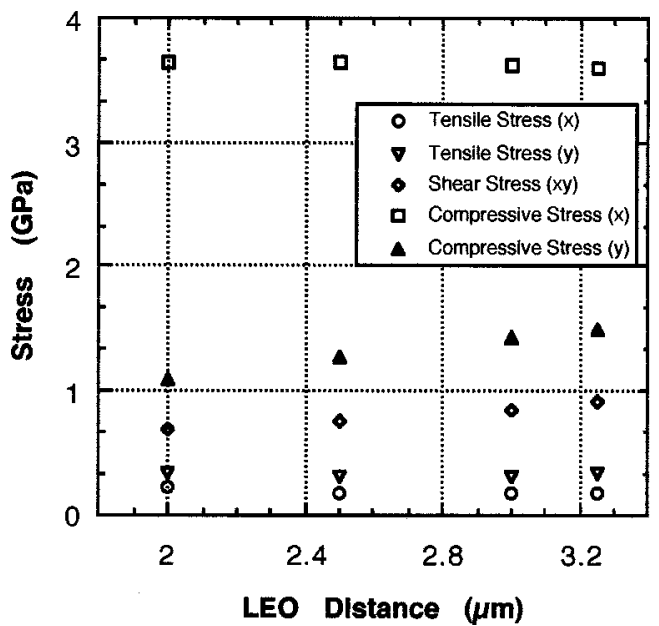

FIG. 4. Maximum tensile, compressive and shear stresses in LEO GaN stripes at the $\mathrm{LEO}-\mathrm{GaN} / \mathrm{SiO}_{2}$ interface as a function of the distance of lateral epitaxial overgrowth on the $\mathrm{SiO}_{2}$ at a fixed window size of $3 \mu \mathrm{m}$ and LEO GaN film thickness of $3 \mu \mathrm{m}$.

Compressive and tensile stresses occur along the $x$ and $y$ directions in the $\mathrm{LEO} \mathrm{GaN}$ adjacent to the $\mathrm{SiO}_{2}$ windows as shown in Figs. 3(a) and 3(b), respectively. These stresses are believed to cause the airfoil shape in the $\mathrm{SiO}_{2}$ and the commonly observed fracture in the LEO GaN either near the window areas at the $\mathrm{GaN} / \mathrm{SiO}_{2}$ interfaces or within the $\mathrm{SiO}_{2}$. An increase in the lateral dimension of the LEO region tends to reduce slightly the thermally generated compressive stresses along the $\mathrm{GaN} / \mathrm{SiO}_{2}$ interface line ( $x$ direction) and increase the compressive stresses along the $\langle 0001\rangle$ direction, as shown in Fig. 4.

Analysis of the shear stress is also important, since the localization of the maximum in this stress likely represents the sites for the onset of plastic relaxation, i.e., dislocations nucleation and propagation and/or film separation. The data presented in Fig. 4 indicate that an increase in the lateral epitaxy region also leads to an increase in the localized maximum shear stresses at the corners of the $\mathrm{LEO} \mathrm{GaN} / \mathrm{SiO}_{2}$ interface. Thus, two keys to achieving defect-free $\mathrm{GaN}$ are: (i) control of the lateral epitaxy and (ii) knowledge regarding the variations in the thermal stress fields in this type of heterostructure.

In summary, TEM studies of selectively and laterally grown $\mathrm{GaN}$ stripes have revealed an airfoil-like morphology of the $\mathrm{SiO}_{2}$ mask layer and void formation within the LEO $\mathrm{GaN}$ layers. FEA of these heterostructures indicated deformation, the deformed shapes likely to be observed and the distribution of the critical stresses. Employment of FEA for the analysis of the stress-strain fields distributions and the corresponding stress gradients within the LEO structure can be utilized to predict and optimize the necessary geometric conditions and the exact geometrical parameters ratios that will result in a structure with the required characteristics in crystal morphology.

The authors acknowledge the support of the Office of Naval Research via Contract No. N00014-96-1-0765 and Cree Research, Inc. for the SiC wafers. The authors gratefully acknowledge help from Jason Griffin with the TEM sample preparation. R. D. acknowledges personal support from the Kobe Steel Ltd. Professorship. T. Z. acknowledges NRC/NAS and Dr. Kenneth A. Jones for ARL for personal support.

${ }^{1}$ S. Nakamura and G. Fasol, The Blue Laser Diode-GaN based Light Emiters and Lasers (Springer, Heidelberg, 1997).

${ }^{2}$ Y. Kato, S. Kitamura, K. Hiramatsu, and N. Sawaki, J. Cryst. Growth 144, 133 (1994).

${ }^{3}$ S. Kitamura, K. Hiramatsu, and N. Sawaki, Jpn. J. Appl. Phys., Part 1 34, 1184 (1995).

${ }^{4}$ R. Underwood, D. Kapolnek, B. Keller, S. Keller, S. DenBaars, and U. Mishra, Topical Workshop on Nitrides, Nagoya, Japan, September, 1995.

${ }^{5}$ R. Underwood, D. Kapolnek, B. Keller, S. Keller, S. DenBaars, and U. Mishra, Solid-State Electron. 41, 243 (1997).

${ }^{6}$ O.-H. Nam, M. Bremser, B. Ward, R. Nemanich, and R. F. Davis, MRS Fall Meeting, Boston, MA, November, 1996.

${ }^{7}$ O.-H. Nam, M. Bremser, B. Ward, R. Nemanich, and R. F. Davis, Jpn. J. Appl. Phys., Part 2 36, L532 (1997).

${ }^{8}$ T. S. Zheleva, O.-H. Nam, M. D. Bremser, and R. F. Davis, Appl. Phys. Lett. 71, 2472 (1997).

${ }^{9}$ O.-H. Nam, T. S. Zheleva, M. D. Bremser, and R. F. Davis, Appl. Phys. Lett. 71, 2638 (1997).

${ }^{10}$ T. S. Zheleva, O.-H. Nam, J. D. Griffin, M. D. Bremser, and R. F. Davis, Mater. Res. Soc. Symp. Proc. 482, 393 (1997).

${ }^{11}$ T. S. Zheleva, O. -H. Nam, W. M. Ashmawi, J. D. Griffin, and R. F. Davis, J. Vac. Sci. Technol. (accepted).

${ }^{12}$ Commercial Code for Finite Element Analysis Ansys 5.3 (1996).

${ }^{13}$ K. Kim, W. Lambrecht, and B. Segal, Phys. Rev. B 53, 16310 (1996).

${ }^{14}$ A. Wright, J. Appl. Phys. 82, 2833 (1997).

${ }^{15}$ CRC Handbook of Chemistry and Physics, 72nd ed., edited by D. Lide (Chemical Rubber Corp., Boca Raton, FL, 1991-1992). 